\title{
Observations in the Saturn system during approach and orbital insertion, with Cassini's visual and infrared mapping spectrometer (VIMS)
}

\author{
R. H. Brown ${ }^{1}$, K. H. Baines ${ }^{2}$, G. Bellucci ${ }^{3}$, B. J. Buratti ${ }^{2}$, F. Capaccioni ${ }^{4}$, P. Cerroni ${ }^{4}$, R. N. Clark ${ }^{5}$, \\ A. Coradini ${ }^{4}$, D. P. Cruikshank ${ }^{6}$, P. Drossart ${ }^{7}$, V. Formisano ${ }^{3}$, R. Jaumann ${ }^{8}$, Y. Langevin ${ }^{9}$, D. L. Matson ${ }^{4}$, \\ T. B. McCord ${ }^{10}$, V. Mennella ${ }^{11}$, R. M. Nelson ${ }^{4}$, P. D. Nicholson ${ }^{12}$, B. Sicardy ${ }^{7}$, C. Sotin ${ }^{13}$, N. Baugh ${ }^{1}$, \\ C. A. Griffith ${ }^{1}$, G. B. Hansen ${ }^{10}$, C. A. Hibbitts ${ }^{10}$, T. W. Momary ${ }^{2}$, and M. R. Showalter ${ }^{6}$
}

1 Department of Planetary Sciences, and Lunar and Planetary Laboratory, University of Arizona, 1629 E. University Boulevard, Tucson, AZ 85721, USA e-mail: rhb@lpl.arizona.edu

2 Jet Propulsion Laboratory, California Institute of Technology, 4800 Oak Grove Boulevard, Pasadena, CA 91109, USA

${ }^{3}$ Consiglio Nazionale delle Ricerche, Isituto di Fisica dello Spazio Interplanetario, Via Fosso del Cavaliere, Tor Vergata, 00133 Roma, Italy

${ }^{4}$ Consiglio Nazionale delle Ricerche, Isituto di Astrofisica Spaziale, via Fosso del Cavaliere, Tor Vergata, 00133 Roma, Italy

5 Mail Stop 964, US Geological Survey, Box 25046, Denver Federal Center, Denver, CO 80225, USA

${ }^{6}$ Mail Stop 245-6, NASA Ames Research Center, Moffett Field, California 94035, USA

7 Observatoire de Paris, Meudon, Department de Recherche Spatial, 5 Place Jules Janssen, 92195 Meudon Cedex, France

8 Institute for Planetary Exploration, Deutsches Zentrum for Luft und Raumfahrt, Rudower Chausee 5, 12489 Berlin, Germany

9 Institute d'Astrophysique Spatiale, Université de Paris, Bâtiment 121, 91405 Orsay Cedex, France

10 Department of Earth and Space Sciences, Johnson Annex A, Box 351310, University of Washington, Seattle WA 98195, USA

11 Istituto Nazionale di Astrofisica, Observatorio Astronomico di Capodimonte, via Moiariello 16, 80131 Napoli, Italy

12 Department of Astronomy, Cornell University, Ithaca, NY 14853, USA

13 Laboratoire de Planetologie et Geodynamique, UMR - CNRS 6112, Faculty des Sciences, BP 92208, 2 rue de la Houssenière, 44072 Nantes Cedex 03, France

Received 13 March 2005 / Accepted 8 August 2005

\section{ABSTRACT}

The Visual and Infrared Mapping Spectrometer observed Phoebe, Iapetus, Titan and Saturn's rings during Cassini's approach and orbital insertion. Phoebe's surface contains water ice, $\mathrm{CO}_{2}$, and ferrous iron. Iapetus contains $\mathrm{CO}_{2}$ and organic materials. Titan's atmosphere shows methane fluorescence, and night-side atmospheric emission that may be $\mathrm{CO}_{2}$ and $\mathrm{CH}_{3} \mathrm{D}$. As determined from cloud motions, the winds at altitude $25-30 \mathrm{~km}$ in the south polar region of Titan appear to be moving in a prograde direction at velocity $\sim 1 \mathrm{~m} \mathrm{~s}^{-1}$. Circular albedo features on Titan's surface, seen at $2.02 \mu \mathrm{m}$, may be palimpsests remaining from the rheological adjustment of ancient impact craters. As such, their long-term persistence is of special interest in view of the expected precipitation of liquids and solids from the atmosphere. Saturn's rings have changed little in their radial structure since the Voyager flybys in the early 1980 s. Spectral absorption bands tentatively attributed to Fe ${ }^{2+}$ suggest that iron-bearing silicates are a source of contamination of the $\mathrm{C}$ ring and the Cassini Division.

Key words. infrared: solar system - planets and satellites: general

\section{Introduction}

This is a preliminary report on observations made with the Cassini Visual and Infrared Mapping Spectrometer (VIMS) (Miller et al. 1996; Reininger et al. 1996) around the time of Saturn orbit insertion (SOI) of the Cassini spacecraft at Saturn, its final destination. Previous reports on VIMS observations of Venus (Baines et al. 2000) and Jupiter and its satellites (Brown et al. 2003; Bellucci et al. 2004;
Chamberlain \& Brown 2004; McCord et al. 2004; Simonelli \& Buratti 2004) made en route to the Saturn system have already appeared. VIMS is an imaging spectrometer capable of obtaining data at 352 separate wavelengths simultaneously, with an angular resolution of $0.17 \times 0.17 \mathrm{mrad}$ over the wavelength region $0.35-1.08 \mu \mathrm{m}$ (the Vis, or visual channel), and an angular resolution of $0.25 \times 0.5 \mathrm{mrad}$ over the range $0.85-5.2 \mu \mathrm{m}$ (the IR, or infrared channel). The Vis and IR channels give spectral resolutions of $7 \mathrm{~nm}$ and $16 \mathrm{~nm}$, respectively. The combined 


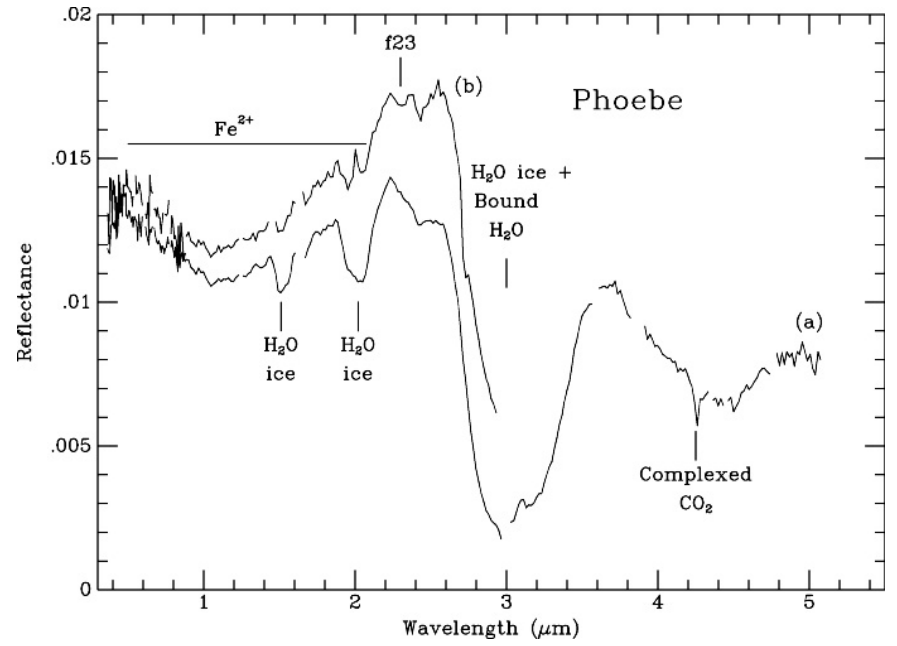

Fig. 1. The spectrum of Phoebe a), consisting of the average of $>10000$ individual spectra and covering the entire surface observed by VIMS. Small gaps in the spectrum correspond to filter gaps and disturbed spectral channels. The small peak at $3.12 \mu \mathrm{m}$ is the Fresnel reflection peak from crystalline $\mathrm{H}_{2} \mathrm{O}$ ice. Some weak spectral absorption bands have received tentative identification; see Clark et al. (2005). Spectrum b) is the average of several pixels in a region of Phoebe in which the metal-OH band (denoted $\mathrm{f} 23$ at $2.3 \mu \mathrm{m}$ ) is particularly strong (spectrum S4 from Fig. 2c of Clark et al. 2005).

wavelength coverage of the Vis and IR channels of VIMS spans about $95 \%$ of the integrated solar irradiance.

\section{Phoebe}

Cassini encountered Phoebe, the outermost large satellite of Saturn, on 11 June 2004. VIMS obtained spatially resolved, hyper-spectral images of Phoebe from 8:47 UT June 11, 2004 at an initial range of $245833 \mathrm{~km}$ and phase angle of $84.9 \mathrm{de}-$ grees, to 10:22 UT June 12, 2004 at a final range of $338401 \mathrm{~km}$ and a phase angle of 92.2 degrees. The closest image was obtained on June 11 at 19:32 UT at a range of $2178 \mathrm{~km}$ and solar phase angle of 24.6 degrees. Figure 1 is a globally averaged spectrum of Phoebe, demonstrating the presence of a number of spectral absorption bands. Noteworthy in this global average are the broad $1 \mu \mathrm{m}$ feature interpreted to be due to $\mathrm{Fe}^{2+}$-bearing minerals, the very strong $3 \mu \mathrm{m}$ band of $\mathrm{H}_{2} \mathrm{O}$ ice, bound water and hydroxyl, and the band at $4.26 \mu \mathrm{m}$ identified as $\mathrm{CO}_{2}$.

The spatial mapping capability of the VIMS produced spatial coverage at full spectral resolution up to $1 \mathrm{~km}$ per pixel. The VIMS data have been registered and projected into three simple cylindrical maps: low resolution providing near global coverage, medium resolution, and a high resolution map of an area of about $2500 \mathrm{~km}^{2}$ (Fig. 2).

The spatially resolved spectra of Phoebe indicate a lowalbedo surface, from $<1$ to $6 \%$ reflectance with a variety of absorption features due to materials which occur with variable abundances and/or grain sizes in different locations on the body (Fig. 2). These include: water ice (previously identified, Owen et al. 1999), bound water, and trapped $\mathrm{CO}_{2}$ (Fig. 2). The broad $1 \mu \mathrm{m} \mathrm{Fe}^{2+}$ feature is present in almost all regions but is more intense in equatorial areas. Bound water and hydroxyl absorptions are indicative of phyllosilicate minerals. Below, we consider each of these constituents of Phoebe's surface in greater detail. See also Clark et al. (2005).

Water ice, identified by its absorption bands at 1.5, 2.0, and $3.0 \mu \mathrm{m}$, is ubiquitous on Phoebe's surface, but generally shows stronger spectral signatures toward the southern polar region (Fig. 2). The highest resolution data indicate an icerich layer exposed in crater walls just below Phoebe's surface, but overall, craters display less exposed ice than does the surrounding terrain (Fig. 2). The cratering events have resulted in relatively ice-poor debris covering exposed ice, in contrast to most craters on Jupiter's $\mathrm{H}_{2} \mathrm{O}$ ice-rich satellites. It is therefore likely that water ice is less abundant in the subsurface, at least in the region of our highest resolution data (near longitude 0 , latitude 0).

The spectral absorption band diagnostic of $\mathrm{Fe}^{2+}$ is broad, extending from $\sim 0.5$ to $2.0 \mu \mathrm{m}$, unlike that in pyroxene mineral phases, but similar to that in olivine and phyllosilicate phases. If phyllosilicate grains were present, weak $\mathrm{OH}$ absorptions would be expected due to the low reflectance. Indeed, weak and relatively narrow 2.2 and $2.3 \mu \mathrm{m}$ absorptions are seen in some spectra, indicating a possible Al-OH absorption. Hydrated mineral absorptions are also consistent with phyllosilicate phases. The 1.5 and $1.95 \mu \mathrm{m}$ hydrated mineral absorptions are at different wavelengths, so a specific mineral cannot be identified. The $2.3 \mu \mathrm{m}$ position matches a number of phyllosilicate phases. In ice-poor regions of Phoebe's surface, these absorption bands can be clearly distinguished from bands of $\mathrm{H}_{2} \mathrm{O}$ ice.

An absorption band attributed to $\mathrm{CO}_{2}$ is seen everywhere on Phoebe covered by the VIMS data, although its strength is variable (Fig. 2). The measured position of the $\mathrm{CO}_{2}$ absorption, $4.260 \mu \mathrm{m}$, is shorter than that of pure $\mathrm{CO}_{2}$ ice $(4.267 \mu \mathrm{m})$. While this wavelength difference is small compared to the intrinsic resolution of the VIMS IR channel, it is statistically significant when seen in several hundred spatial pixels. The observed wavelength on Phoebe is similar to that of the trapped $\mathrm{CO}_{2}$ found in the $\mathrm{H}_{2} \mathrm{O}$ ice-poor regions of Jupiter's satellites Callisto and Ganymede (McCord et al. 1997, 1998; Hibbitts et al. 2002, 2003). The VIMS data with the highest spatial resolution on Phoebe indicate a linear surface structure at least $60 \mathrm{~km}$ in length where $\mathrm{CO}_{2}$ absorption is weak, and variable $\mathrm{CO}_{2}$ abundances are also seen in medium-resolution spectral images.

In summary, spectra of Phoebe indicate ferrous ironbearing minerals, bound water, carbon dioxide, and probable phyllosilicate phases. These materials exist in distinct locations on Phoebe's surface, with general polar versus equatorial spatial variations. While the overall composition of Phoebe is similar to comets, there is some evidence that ice may only be a surface coating. This suggests that Phoebe is coated by material of cometary or outer Solar System origin, but this information alone is insufficient to allow the conclusion that Phoebe itself originated in the outer Solar System. The same broad $\mathrm{Fe}^{2+}$ absorption is seen in Saturn's rings, in particular, in the Cassini Division and in the $\mathrm{C}$ ring. This implies a common material on Phoebe's surface and the rings. The detection of an absorption band of uncertain origin at 


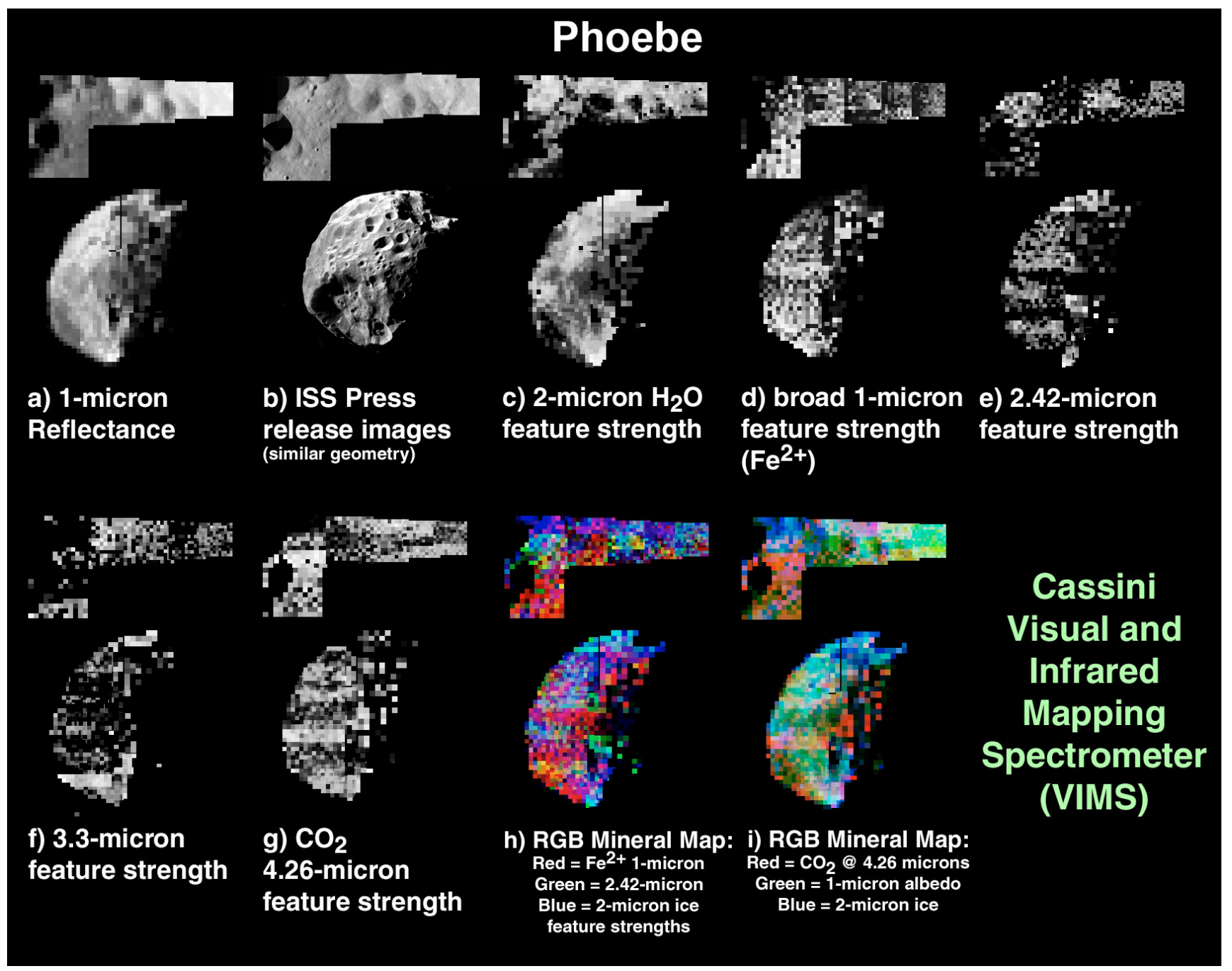

Fig. 2. Compositional maps of Phoebe in simple cylindrical projection. For each map, two resolutions are shown: low resolution sampled at 1 degree per pixel (about $3.8 \mathrm{~km}$ at the equator) and high resolution sampled at $0.96 \mathrm{~km} /$ pixel. Also see Clark et al. (2005).

$2.42 \mu \mathrm{m}$ on both Phoebe and Iapetus (see next section) may indicate that material from Phoebe has coated Iapetus's leading hemisphere. Alternatively, perhaps cometary material has coated both Phoebe and Iapetus. Regardless of their origin, Phoebe's surface materials are unique among Solar System surfaces observed to date and probably sample primitive materials that originated in the region well beyond Jupiter.

\section{3. lapetus}

Saturn's large satellite Iapetus has long been known for its unique bimodal surface albedo distribution. The satellite is locked in synchronous rotation relative to Saturn, the leading hemisphere being characterized by a low albedo $(\sim 6 \%)$ and distinctly reddish color. The opposite, or trailing, hemisphere has an albedo of about $60 \%$, and displays strong absorption bands of $\mathrm{H}_{2} \mathrm{O}$ ice (Clark et al. 1986). The origin and composition of the low-albedo material on the leading hemisphere have been vexing problems for many years (Owen et al. 2001; Grundy et al. 1999).
Cassini encountered Iapetus on 31 December 2004. VIMS obtained spatially resolved, hyperspectral images over a range of distances and phase angles. The mean values of range (in thousands of $\mathrm{km}$ ) and phase angle, respectively, for the four VIMS cubes near closest approach are: LIMBTOPO B 146.5, 63.5 ${ }^{\circ}$, LIMBTOPO D 124, 93.5 ${ }^{\circ}$, LIMBTOPO E $126.5,105^{\circ}$, GLOBMAP C $128.5,79.5^{\circ}$. An average spectrum of a portion of the high-albedo hemisphere is shown in Fig. 3, together with the grand average spectrum of the low-albedo hemisphere. Projected on the surface of Iapetus, the $0.5 \mathrm{mrad}$ pixels of the VIMS infrared cubes correspond to $73 \mathrm{~km}$ at a range of $146500 \mathrm{~km}$, and $62 \mathrm{~km}$ at a range of $126000 \mathrm{~km}$.

The spectrum of the low-albedo hemisphere (Buratti et al. 2005 ) is similar to that obtained from Earth-based telescopes, but extends to longer wavelengths. A strong $3 \mu \mathrm{m}$ band is confirmed; models of the low-albedo hemisphere (Owen et al. 2001) favor a nitrogen-rich tholin as the source of the red color of the surface, as well as the strong $3 \mu \mathrm{m}$ absorption band. In the present paper, the $3 \mu \mathrm{m}$ band is attributed to bound water, presumably in the dark material itself. While the bright, $\mathrm{H}_{2} \mathrm{O}$ ice-rich region of Iapetus is deeply absorbing between 4 and 


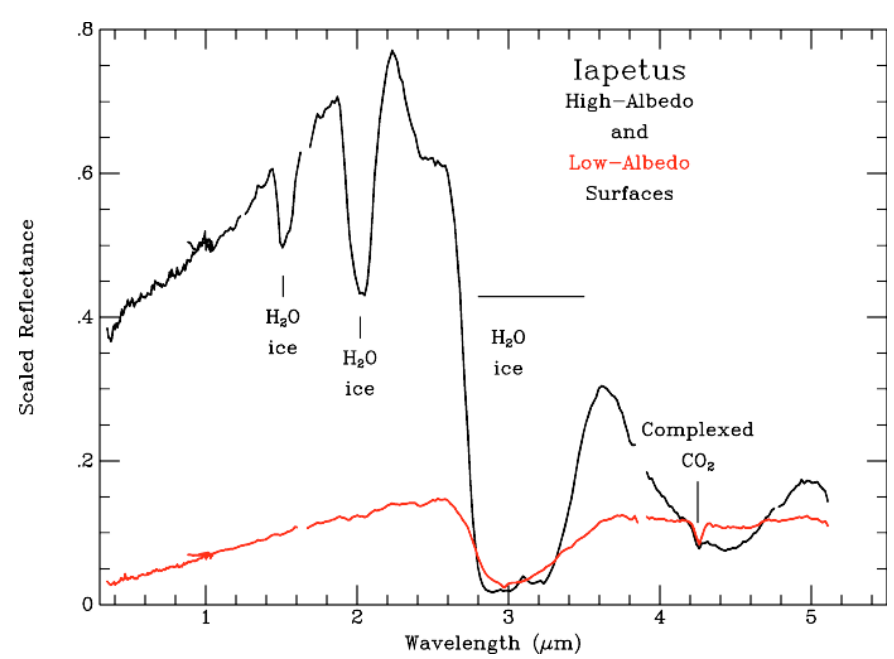

Fig. 3. The average spectrum of a portion of the high-albedo surface of Iapetus, observed at the close flyby of December 31, 2004, at phase angle $\sim 90^{\circ}$. Small gaps in the spectrum correspond to filter gaps and disturbed spectral channels. The small peak at $3.12 \mu \mathrm{m}$ is the Fresnel reflection peak from crystalline $\mathrm{H}_{2} \mathrm{O}$ ice. The only materials identified in this spectrum are the bands of $\mathrm{H}_{2} \mathrm{O}$ ice and complexed $\mathrm{CO}_{2}$ $(4.26 \mu \mathrm{m})$. Additional discussion is found in Buratti et al. (2005). The spectrum of the low-albedo hemisphere shows the broad $3 \mu \mathrm{m}$ band attributed to bound $\mathrm{H}_{2} \mathrm{O}$, the $4.26 \mu \mathrm{m}$ band of complexed $\mathrm{CO}_{2}$, and several weaker, unidentified features. The two spectra are scaled in intensity such that at $2.2 \mu \mathrm{m}$ the reflectance of the high-albedo hemisphere is 0.76 and that of the low-albedo hemisphere is 0.14 . These reflectance values are the equivalent of geometric albedo at $2.2 \mu \mathrm{m}$ at small phase angles observable from Earth (e.g., Owen et al. 2001).

$5 \mu \mathrm{m}$, the low-albedo side of Iapetus has about the same reflectivity in this spectral range as it does at $2.2 \mu \mathrm{m}$. This result is consistent with the presence of abundant organic material, such as N-rich tholins, which are highly reflective between 4 and $5 \mu \mathrm{m}$, as shown in the Iapetus models of Owen et al. (2001, Fig. 13) and Buratti et al. (2005).

A prominent, newly recognized feature in spectra of both the high- and low-albedo material on Iapetus is an absorption band at $4.255 \mu \mathrm{m}$, which we identify as the $v_{3}$ antisymmetric stretching mode of $\mathrm{CO}_{2}$ adsorbed on or included in another material (Fig. 3). As in the case of Phoebe, the $\mathrm{CO}_{2}$ band is shifted to shorter wavelengths than that of $\mathrm{CO}_{2}$ ice, which in any case would not be stable on Iapetus because of the relatively high temperature. At the limited spatial scale afforded by these data, we can say that the $\mathrm{CO}_{2}$ band on Iapetus occurs in regions that are $\mathrm{H}_{2} \mathrm{O}$ ice-poor, but not ice-free, as is also true in the case of Phoebe. This circumstance allows for the possibility that $\mathrm{CO}_{2}$ and $\mathrm{H}_{2} \mathrm{O}$ are associated (complexed) in a way that induces the wavelength shift observed for the $\mathrm{CO}_{2}$. An alternative possibility is the presence of $\mathrm{CO}_{2}$ gaseous or fluid inclusions in minerals (McCord et al. 1998).

In addition to the strong $3 \mu \mathrm{m}$ bound $\mathrm{H}_{2} \mathrm{O}$ band and the $4.26 \mu \mathrm{m}$ band of complexed $\mathrm{CO}_{2}$, several relatively weak absorptions appear in the spectrum of the low-albedo hemisphere. The band at $2.418 \mu \mathrm{m}$ is notable because it also appears in the spectrum of Phoebe; Clark et al. (2005) propose that it is an overtone of a $\mathrm{CN}$-bearing mineral. Additional absorptions likely due to $\mathrm{CN}$-bearing minerals occur in the region 4.6-4.8 $\mu \mathrm{m}$.

Additional absorption bands in the spectra of both the low and high-albedo materials of Iapetus require further study and modelling for identification and a more complete understanding of the way in which these materials are mixed with one another.

\section{Titan: atmosphere}

In addition to the well known atmospheric absorption bands of $\mathrm{CH}_{4}$ and $\mathrm{CO}$, the observations of Titan by VIMS show prominent limb emission in the $\mathrm{CH}_{4}$ bands near $3.3 \mu \mathrm{m}$. While methane emission was detected on the full disk from Earthbased observations (Kim et al. 2000; Geballe et al. 2003), the VIMS observations have a spatial resolution of $\sim 135 \mathrm{~km}$, which is sufficiently high to resolve the limb emission and show that the maximum of the emitted radiation occurs at an altitude of about $400 \mathrm{~km}$ (Fig. 4). A synthetic model used to estimate methane abundances from fluorescent emission on giant planets (Drossart et al. 1999) has been adapted to allow an estimate of the abundance of methane in the atmosphere of Titan. In this model, non-LTE emission is treated with a full radiative transfer, line-by-line frequency redistribution calculation. Solar absorption is calculated in all the methane bands up to the $1.7 \mu \mathrm{m}$ bands, and cascade fluorescence is retrieved for the fundamental ( $v_{3}$ band, $\left.3.313 \mu \mathrm{m}\right)$, the first overtone $\left(v_{3}+v_{4}-v_{4}\right.$, $3.326 \mu \mathrm{m})$, the second overtone $\left(2 v_{3}-v_{3}, 3.35 \mu \mathrm{m}\right)$, and related bands at similar wavelengths. The vibrational population is calculated in a simplified scheme ("superlevels"), following Doyennette et al. (1998). Self-absorption and subsequent scattering in the Titan atmosphere is treated by a Curtis matrix method. The calculations are made in full spherical geometry for different altitudes of sounding along a tangential path, and for the solar phase angle corresponding to image A in Fig. 4.

The model of fluorescence has been applied with different values of the $\mathrm{CH}_{4}$ abundance (from 1 to $3 \%$ ) with an acceptable fit to the observed spectra; even if the mixing ratio of $\mathrm{CH}_{4}$ could possibly be retrieved from a complete calculation, the uncertainties in the modelling, in particular for the collision frequency (no less than $50 \%$ according to different authors), preclude an accurate direct determination of $\mathrm{CH}_{4}$ from this set of observations. Nevertheless, relative variations of $\mathrm{CH}_{4}$ abundance would be detectable; the uniformity of the pole-to-equator emission of $\mathrm{CH}_{4}$ allows us to deduce a constant abundance of $\mathrm{CH}_{4}$ within $20 \%$.

The spectral fit depends on the abundance of methane in the stratosphere, assumed to be constant over the sounded levels (200 to $800 \mathrm{~km}$ above the surface). Uncertainties in the model arise from several sources: the spectral calibration of VIMS gives an uncertainty in the retrieved flux of about $20 \%$, the atmospheric model (15\%), the accuracy of vibrational parameters (50\%) (Boursier et al. 2003), and the accuracy of the complex model calculations $(>10 \%)$. From the model, we estimate an abundance of methane of $2 \%$ (number density) with an accuracy of $50 \%$.

Spectral emission on the night side of Titan is also seen in the VIMS data (Fig. 5). In addition to an overall spectral 

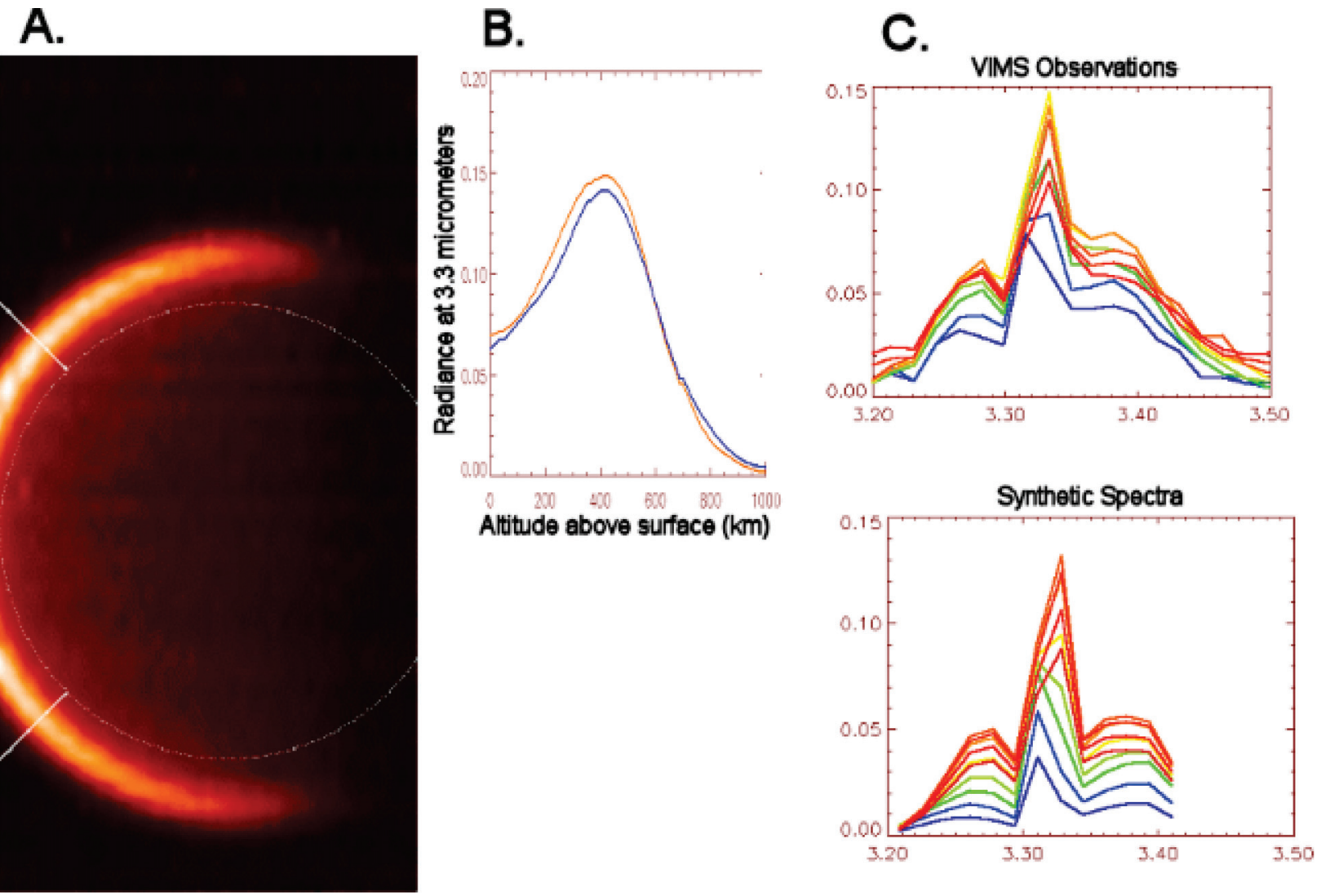

Fig. 4. A. Image of Titan at high phase angle with VIMS, showing the size of the body (circle) and two radial lines at which spectral profiles were measured at wavelength $3.3 \mu \mathrm{m}^{-1}$. B. Profiles of radiance along the two radial segments shown in image A. The units of radiance are $\mu \mathrm{W} \mathrm{cm}{ }^{-2} \mathrm{Sr}^{-1} \mu \mathrm{m}^{-1}$. C. A series of spectra (top panel) corresponding to different positions along the radial line segments of image $\mathbf{A}$, compared with synthetic spectra (lower panel) calculated for the same geometry. These observations were obtained at the T0 flyby (VIMS cube CM_1467450817) on 2004 July 2 at 08:53 UT from a distance of $339750 \mathrm{~km}$, with a phase angle of $65^{\circ}$ and a spatial pixel size of $\sim 135 \mathrm{~km}$ at the center of disk.

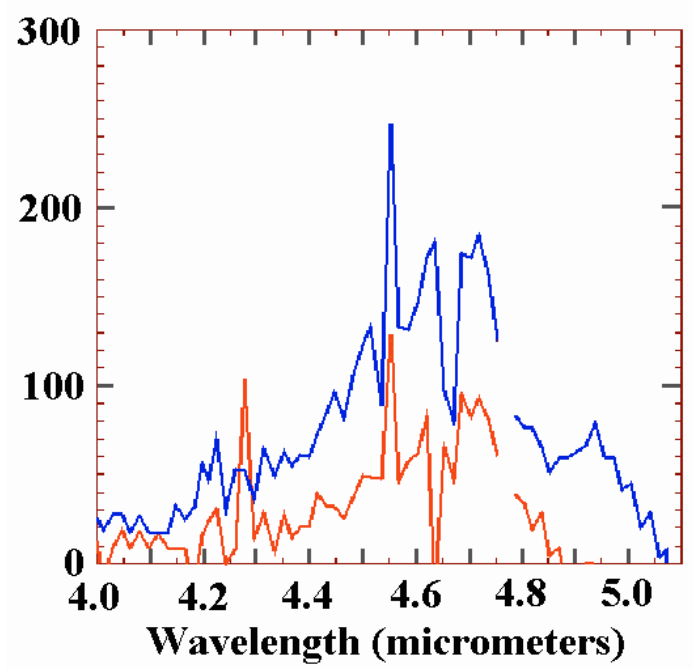

Fig. 5. Spectra of the night side of Titan from the same VIMS cube described in the caption to Fig. 4. Blue is for the limb and red is for the center of the disk.

shape modulated by $\mathrm{CO}$ thermal emission, emission features are observed which could be due to $\mathrm{CO}_{2}$ at $4.3 \mu \mathrm{m}$ (prominent on the disk) and $\mathrm{CH}_{3} \mathrm{D}$ at $4.55 \mu \mathrm{m}$ (prominent at the limb).
The interpretations of these spectra are complicated by nonLTE effects, which complicate the radiative transfer calculations, and will be the subject of further investigation.

In the $5 \mu \mathrm{m}$ region, $\mathrm{CO}$ fluorescence has been detected on Titan from ground-based observations with the ISAAC spectrometer on the Very Large Telescope (López-Valverde et al. 2005). Despite its lower spectral resolution, VIMS is sensitive enough to detect the $\mathrm{CO}$ emission, with additional observations in thermal emission on the night side.

The Titan encounter of 2 July 2004 provided the first opportunity for VIMS to measure winds on Titan by tracking discrete clouds. Spectral imagery revealed that cloud coverage of Titan was sparse, amounting to less than $1.5 \%$ of the observed sunlit surface area. Nevertheless three distinct clouds at an estimated altitude of $25-30 \mathrm{~km}$ above the surface near the South Pole were followed during the encounter (Fig. 6). The variable brightness of the cloud features over our 13-h observational period is an indicator of small-scale meteorological activity which hinders our ability to measure advective motions. Here we report results of the measurements of the centroid of each of the three features as an indicator of mean dynamical motion, possibly due to a variety of mechanisms, including wave activity as well as advection. All of the measured motions were slow (less than $4 \mathrm{~m} / \mathrm{s}$ ) and the clouds moved mainly 
in a zonal direction. The most prominent cloud (a roughly circular feature $600 \pm 110 \mathrm{~km}$ in diameter) was located near the South Pole $\left(\sim 87^{\circ}\right.$ south latitude, $\sim 0^{\circ}$ longitude). Cloud motions were measured using two methods. In the first, cloud positions were measured in latitude/longitude coordinates using the postencounter SPICE kernels supplied by the Cassini Project, and in the second the relative position of the centroid of each cloud was measured against prominent surface features observed by VIMS in the clear $2.02 \mu \mathrm{m}$ channel (Fig. 6). Both methods showed that the clouds remained nearly stationary during the encounter, but a careful analysis revealed a mean zonal wind speed over a 13 -h period of $0.5 \pm 3.3 \mathrm{~m} \mathrm{~s}^{-1}$ in the prograde direction. Two other smaller clouds located within $20^{\circ}$ of the South Pole also showed sluggish movements during this 13-h period. The zonal velocity components were $0.9 \pm 3.0$ and $2.3 \pm 2.0 \mathrm{~m} \mathrm{~s}^{-1}$ for clouds at $74^{\circ}$ and $78^{\circ}$ south latitude, respectively, in a prograde direction. While the uncertainties in these cloud measurements allow for retrograde or no motion, the totality of all the evidence favors a weak prograde motion, consistent with the results of other investigators using groundbased images (e.g., Bouchez \& Brown 2005). The mean meridional motions during the 13 -h period were imperceptible for all other clouds.

\section{Titan: surface}

Sunlight penetrates Titan's atmosphere at wavelengths (e.g., $2.02 \mu \mathrm{m}$ ) where $\mathrm{CH}_{4}$, the principal atmospheric gaseous absorber, is transparent. At such wavelengths, VIMS is able to image the surface (Fig. 7). We investigated the possibility that several circular features detected in VIMS images of the Titan's surface might be impact craters (or impact remnants). Over geologic time impact craters on an ice-rich planetary surface may relax due to plastic flow of the lithosphere (or cryosphere), resulting in reduced or non-existent topographic relief. These features may remain visible as palimpsests - regions of different reflectance on a surface having little or no topographic variation. In the absence of shadows or other clear signs of vertical relief, we undertook a photometric analysis of two of the circular features that were imaged in order to search for some degree of topographic expression. The center of the first feature studied was at latitude $-36^{\circ}$ and longitude $31^{\circ}$, and the center of the second was at latitude $-7^{\circ}$, longitude $357^{\circ}$. We used the VIMS image at $2.02 \mu \mathrm{m}$ and measured the reflectance along lines which pass through the sub-solar point on Titan's surface and traverse the center of the circular features (Fig. 7). We compared our photometric traces with the profiles expected from four different cases: 1) a simple circular depression; 2) a circular depression with a raised rim; 3) a circular depression with a raised rim and a central peak; and 4) a circular depression with a raised rim and a central pit. We assumed that surface particles scatter incident sunlight isotropically with a uniform single scattering albedo, and we applied the widely used bi-directional reflectance equations (Hapke 1981).

Under these assumptions we could only reconstruct the photometric measurements by assuming depressions of unreasonable depth $(>50 \mathrm{~km}$.). Therefore, we conclude that the circular features are due to differences in surface reflectance.
However, a possible impact origin cannot be ruled out because these features might be palimpsests resulting from the relaxation of the original topography by plastic flow. If these features are indeed palimpsests representing the remains of ancient impacts, their persistence on the surface suggests that weathering processes on Titan are limited. We note furthermore that because these features are defined primarily by surface albedo variations, the degree of global precipitation of either solids or liquids from the atmosphere, and the hypothesized liquid oceans are limited.

The reflectance spectrum of the surface of Titan in the nearinfrared is difficult to derive because of the absorbing and scattering effects of the methane- and aerosol-rich atmosphere, but at wavelengths where methane absorption is minimum $(0.83$, $0.94,1.07,1.28,1.58,2.0,2.9$, and $5.0 \mu \mathrm{m})$, radiative transfer models can remove most of the interference (Griffith et al. 2003). Work is in progress to model the surface reflectance of Titan with the VIMS data, and will be reported elsewhere (McCord et al., in preparation).

\section{Rings}

Immediately following the Cassini spacecraft's Saturn Orbit Insertion (SOI) engine burn on 1 July 2004, VIMS obtained near-infrared spectra from 0.9 to $5.1 \mu \mathrm{m}$ in two continuous radial scans across the unlit side of the rings, at ranges of 24000 to $38000 \mathrm{~km}$. The first scan covers the outer $\mathrm{C}$ and inner $\mathrm{B}$ rings at a phase angle $\alpha=82^{\circ}$ and an emission angle $\varepsilon=47^{\circ}$, while the second covers the Cassini Division and entire A ring at $\alpha=59^{\circ}$ and $\varepsilon=63^{\circ}$. The solar incidence angle was $114^{\circ}$ and the radial resolution ranges from 15 to $25 \mathrm{~km}$ per pixel, with sampling intervals of $2.5 \mathrm{~km}$ in the $\mathrm{C}$ ring and $\sim 7 \mathrm{~km}$ in the $\mathrm{A}$ ring. The IR integration time was $160 \mathrm{msec}$ per pixel.

Structurally, the rings appear to have changed little, if at all, since the Voyager observations in 1980/81 and the occultations by the star 28 Sagittarius (Sgr) in 1989 (Nicholson et al. 2000). In the outer $\mathrm{C}$ ring, the familiar pattern of moderate optical depth "plateaux" is seen, and the only variable feature is the eccentric Maxwell ringlet (Porco et al. 1984), which was sampled by VIMS close to pericenter at a radius of $87477 \pm 7 \mathrm{~km}$. This is consistent with a precessing Keplerian ellipse model fitted to the Voyager and 28 Sgr occultation data ${ }^{1}$.

At most locations in the moderately opaque inner B ring there is a strong anticorrelation between the transmitted intensity and the normal optical depth, as anticipated in this viewing geometry. Particularly striking is the long-term stability of the quasi-irregular structure which characterizes this region on scales of $\sim 100 \mathrm{~km}$ (Fig. 8). The origin of this structure is unknown (Horn \& Cuzzi 1996), but theories have invoked several varieties of spontaneous radial instability (Ward 1981; Linn \& Bodenheimer 1981; Borderies et al. 1985;

\footnotetext{
1 Unpublished 1991 model by G. Musser, P. D. Nicholson, and R. G. French, with keplerian parameters $a=87512 \pm 4 \mathrm{~km}, e=$ $(4.4 \pm 0.5) \times 10^{4}, w_{\mathrm{o}}=240 \pm 4^{\circ}$ at JD 2444556.49 , and $\mathrm{d} w / \mathrm{d} t=$ $14.6935 \pm 0.0017^{\circ} / \mathrm{d}$. This model predicts a true anomaly for the SOI observations of $\lambda-w=322 \pm 15^{\circ}$, corresponding to a radius of $87483 \pm 6 \mathrm{~km}$.
} 


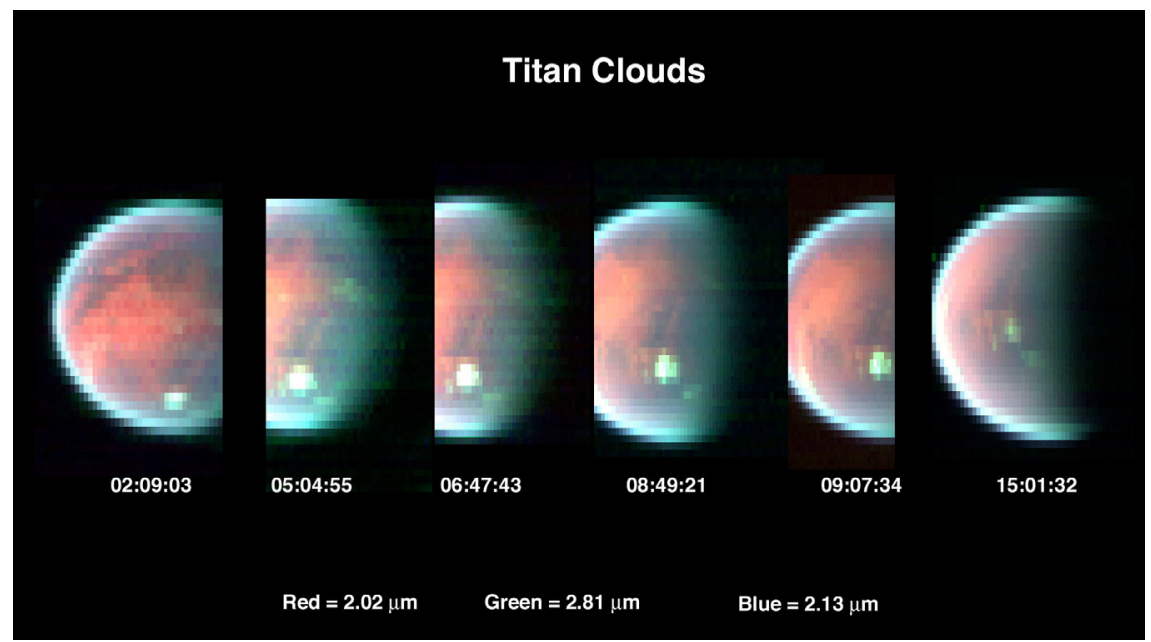

Fig. 6. Clouds and surface features as observed in six near-infrared false-color views of Titan over a 12-h period, July 2, 2004. These images were acquired from a distance of $\sim 350000 \mathrm{~km}$. In each image, red and green depict the views at 2.01 and $2.81 \mu \mathrm{m}$ (respectively); the atmospheric "window" wavelengths in which sunlight reaches the surface relatively unimpeded by atmospheric absorption and aerosol extinction. By contrast, the blue represents $2.13 \mu \mathrm{m}$, a wavelength largely attenuated by the lower atmosphere. High-altitude clouds and stratospheric haze layers appear bluish-white on the disk and limb, since they reflect substantial sunlight at all three wavelengths. Discrete cloud features appear most prominently near the South Pole in all of these images, and their motion allows estimates of the wind speeds at cloud-top altitudes. Note the changing perspective over the 13-h period, as the spacecraft flew nearly directly over the South Pole.

Goertz \& Morfill 1988; Schmit \& Tscharnuter 1999; Tremaine 2003) or ballistic transport (Durisen et al. 1989; Durisen 1995).

Spectrally, all regions of the rings scanned are dominated by water ice, with prominent absorption bands at 1.55, 2.0 and $3.0 \mu \mathrm{m}$, as well as weaker bands at 1.04 and $1.25 \mu \mathrm{m}$ which are seen primarily in the A and B rings (Fig. 9). All ice bands are strongest in the middle $\mathrm{A}$ ring, around $131000 \mathrm{~km}$ radius, and the outer $\mathrm{B}$ ring, and much weaker in the $\mathrm{C}$ ring and Cassini Division (Fig. 10). This broad picture is generally consistent with Earth-based reflectance spectra at higher spectral resolution obtained at low phase angle, except that the Earth-based spectra show stronger 1.04 and $1.25 \mu \mathrm{m}$ bands in the $\mathrm{C}$ ring (Poulet et al. 2003). Systematic increases in band strength with radius are observed for the 1.25 and $2.0 \mu \mathrm{m}$ bands across the outer $\mathrm{C}$ ring and into the innermost $\mathrm{B}$ ring, and across the inner Cassini Division into the inner A ring. The water ice bands weaken again in the outer A ring, especially outside of the Keeler gap.

Regionally, the transitions between the $\mathrm{C}$ and $\mathrm{B}$ rings and between the Cassini Division and A ring, which are quite abrupt in optical depth, are marked by gradual changes in ice band depth over radial distances of a few thousand $\mathrm{km}$, perhaps indicative of ballistic redistribution of material (Durisen et al. 1992; Cuzzi et al. 2003). Locally, however, the fractional ice band depths are almost independent of optical depth in both the $\mathrm{C}$ and $\mathrm{B}$ rings, as indicated by the lack of a significant correlation between small-scale brightness features and band depths (Fig. 10). This suggests that the light diffusely transmitted through the rings at moderate phase angles is dominated by single scattering from the centimeter-to-meter sized ring particles, although the band shapes and relative strengths imply multiple scattering between the ice grains on the surfaces of these particles. A similar lack of correlation with the underlying small-scale optical depth variations in the $\mathrm{C}$ ring is seen for

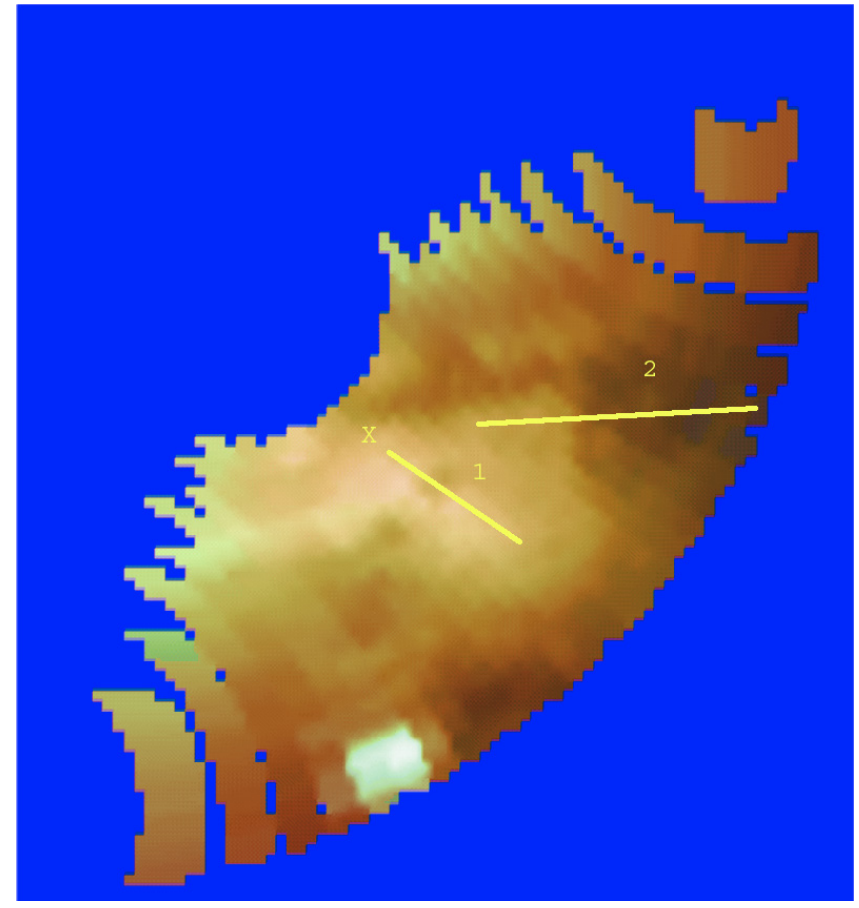

Fig. 7. Color composite image developed from three VIMS spectral images at wavelengths of $1.27,2.02$, and $2.78 \mu \mathrm{m}$, three wavelengths at which methane, Titan's principal atmospheric absorber, is transparent. There are several circular features resembling impact craters, two of which we investigated in detail. The subsolar point is indicated by the letter X. Photometric profiles were measured along the lines show passing through the center of each of the two circular features that we studied and in line with the sub solar point.

the optical green to violet color ratios in reflected light (Estrada et al. 2003). 


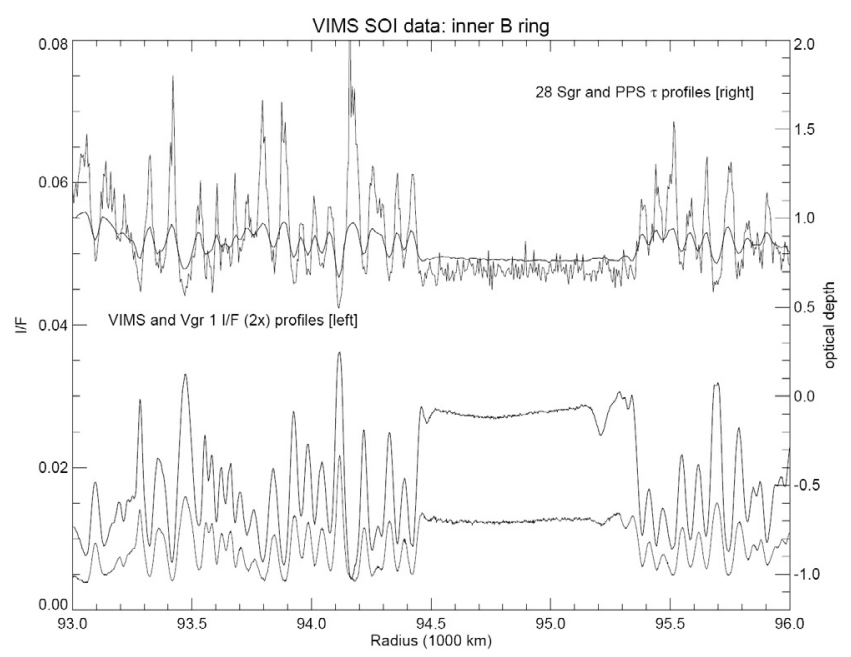

Fig. 8. The lower two curves show $\mathrm{I} / \mathrm{F}$ profiles of the inner B ring as seen in diffuse transmission, from Voyager 1 narrow angle camera images (wavelength $0.45 \mu \mathrm{m}$, thin line) taken in 1980 and CassiniVIMS SOI observations $(1.08 \mu \mathrm{m}$, thick line) in 2004. The upper curves show profiles of normal optical depth for the same region, as measured by the Voyager 2 Photopolarimeter occultation (wavelength $0.27 \mu \mathrm{m}$, thin line) in 1981 and the $28 \mathrm{Sgr}$ stellar occultation $(3.9 \mu \mathrm{m}$, thick line) in 1989. No significant changes in ring structure are seen over this 24 year interval. As expected, there is a strong anticorrelation between I/F and optical depth in this dark-side viewing geometry.

Upon closer inspection of Fig. 10, however, it can be seen that the 1.25 and $2.0 \mu \mathrm{m}$ ice bands are slightly (10\%) stronger in the higher-optical depth "plateaux" in the outermost $\mathrm{C}$ ring than in the intervening regions, whereas in the part of the B ring seen in Fig. 8 there is a weak anticorrelation between band strength and optical depth. In the A ring, small-scale variations in ice band strength are associated with the strong Lindblad resonances due to Janus (4:3, 5:4 and 6:5) and Mimas (5:3). In each case, we see stronger bands over a region of width $\sim 200 \mathrm{~km}$ which corresponds to the density wave driven outwards from the resonance (Holberg et al. 1982), flanked on either side by regions of weaker than average band strength which extend over a total width of $\sim 750 \mathrm{~km}$. Curiously, the Mimas 5:3 vertical resonance and associated bending wave (Lissauer 1985) show no comparable spectral signature.

A broad reflectance maximum at $3.6 \mu \mathrm{m}$, characteristic of ice grain sizes less than $100 \mu \mathrm{m}$, is prominent everywhere in the SOI scans but particularly strong in the outermost parts of the A ring, exterior to the Encke Gap (Fig. 10). The $3.12 \mu \mathrm{m}$ Fresnel peak due to front-surface reflection from (large?) ice grains is clearly observed in the A ring, especially in its outermost parts, but not in the $\mathrm{B}$ and $\mathrm{C}$ rings (Fig. 10). This feature is seen in reflected light in both $\mathrm{A}$ and $\mathrm{B}$ rings in pre-SOI VIMS spectra at a phase angle of $\sim 60^{\circ}$ and in ground-based spectra at $5^{\circ}$ phase (Poulet et al. 2003). Variations in the 3.1 and $3.6 \mu \mathrm{m}$ peaks, both spatially and with viewing geometry, should eventually provide information on ice grain sizes and regolith texture on the surfaces of ring particles, but this must await further observations at a wider range of geometries.

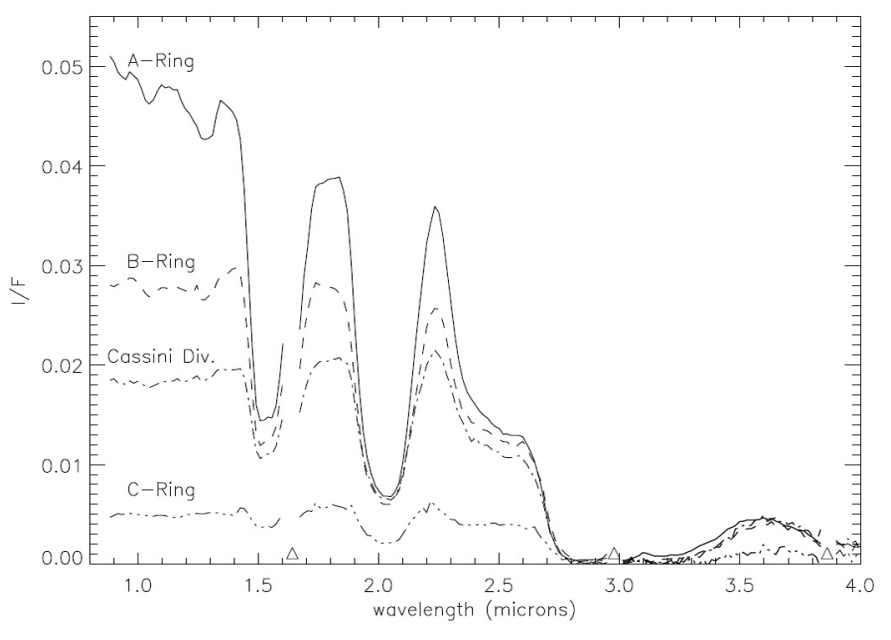

Fig. 9. Spectra of the major ring regions as seen in the VIMS SOI data, averaged over radial ranges of $126-130 \mathrm{Mm}$ for the A ring, 119.0-119.3 Mm for the Cassini Division, 94.6-95.1 Mm for the $\mathrm{B}$ ring and $87.7-88.2 \mathrm{Mm}$ for the $\mathrm{C}$ ring. Triangles denote the locations of gaps between blocking filters in the VIMS focal plane array, where the spectrum is unreliable. At an exposure time of $160 \mathrm{~ms}$ per pixel, there is no useful signal beyond $\sim 4.0 \mu \mathrm{m}$. Noteworthy are the prominent water ice bands at 1.04, 1.25, 1.5, 2.0 and $2.9 \mu \mathrm{m}$. The blue continuum slopes in the A and B ring between 0.9 and $2.2 \mu \mathrm{m}$, characteristic of pure water ice, contrast with the neutral to red continuum slopes shown by the $\mathrm{C}$ ring and Cassini Division, which we attribute to absorption by $\mathrm{Fe}^{2+}$.

Besides water ice, the most noteworthy spectral feature in the SOI scan of the rings is a broad, shallow absorption in the $0.9-1.4 \mu \mathrm{m}$ region which we tentatively attribute to Fe-bearing minerals, most likely silicates. This feature is similar to that seen on Phoebe as described above, and is seen primarily in the outer $\mathrm{C}$ ring, and the Cassini Division, and possibly also at the edges of the Encke and Keeler gaps and the very outer edge of the A ring (Fig. 10). However, like the ice bands, it varies in strength smoothly across structural boundaries. It may also be responsible for the red slope of the continuum noted by Poulet et al. (2003) in their $\mathrm{C}$ ring spectra, compared to those of the $\mathrm{A}$ and $\mathrm{B}$ rings, and attributed by them to an unknown dark, spectrally-neutral contaminant which they modeled as amorphous carbon inclusions within the ice grains. Cuzzi \& Estrada (1998) have suggested that the lower visual albedo and less-red visual color of the $\mathrm{C}$ ring and Cassini Division particles can be understood as a natural result of bombardment of an initially pristine icy ring system by spectrally-neutral material of interplanetary origin. This model also appears to be compatible with the VIMS data, although the visual and near-IR colors may be controlled by distinct materials.

Poulet et al. (2003) reported the presence of absorption features at 1.73 and $3.4 \mu \mathrm{m}$ which are probably due to hydrocarbons ( $\mathrm{CH}$ bonds) in the $\mathrm{C}$ ring. Similar features are also seen in the innermost portion of the VIMS $\mathrm{C}$ ring scan, accompanied by the weaker $\mathrm{CH}$ bands at 1.00, 1.16, 1.38 and $2.20 \mu \mathrm{m}$ as well as the stronger $2.3 \mu \mathrm{m}$ band. However, the $\mathrm{C}$ ring SOI scan was made immediately adjacent to the shadow cast by Saturn across the rings and based on subsequent observations 


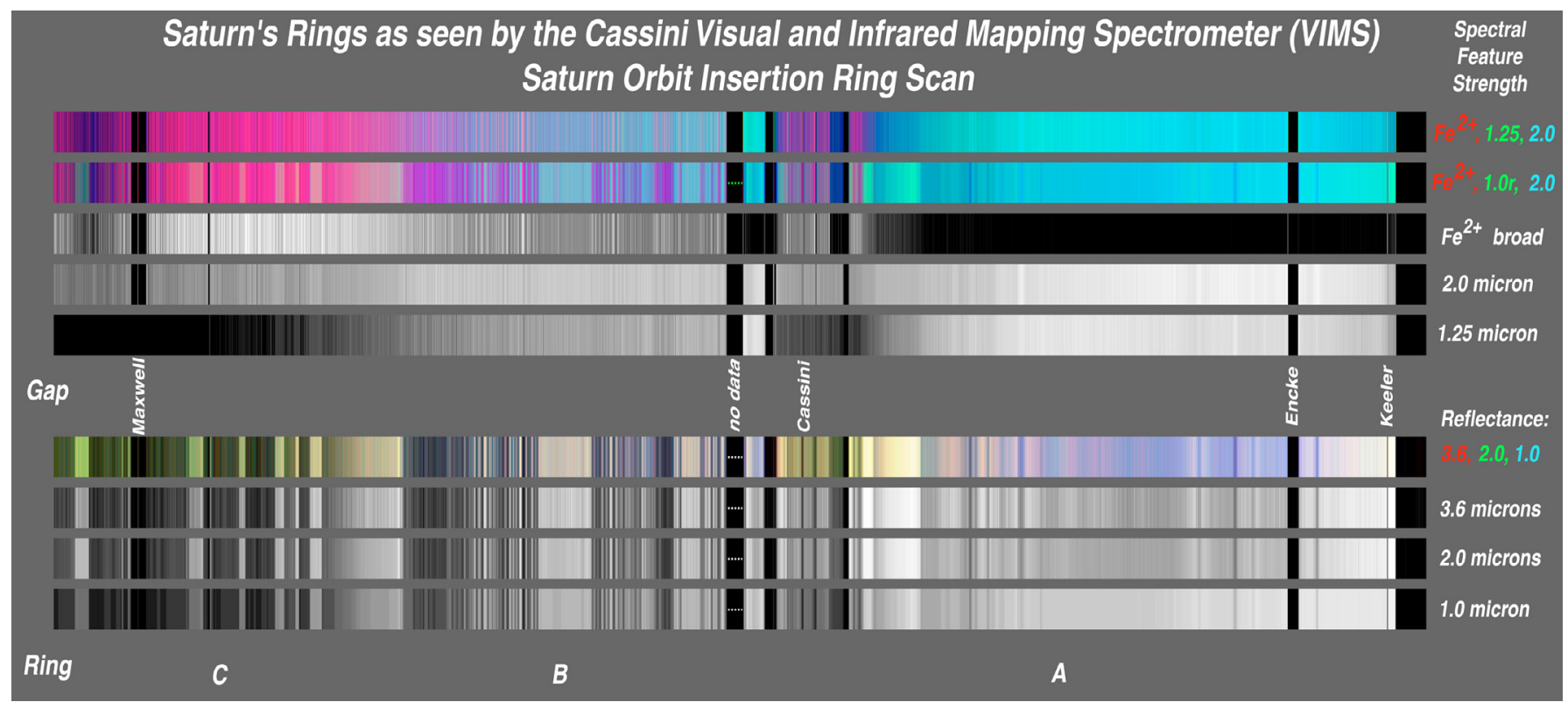

Fig. 10. Map of Saturn's rings as seen in diffuse transmission in the Cassini VIMS SOI scans. The horizontal scale is approximately linear in radius, with the major ring regions identified at the bottom of the diagram. The $\mathrm{C} / \mathrm{B}$ ring scan is shown at its original sampling of $2.5 \mathrm{~km}$, while the Cassini Division/A ring scan has been interpolated onto a $5 \mathrm{~km}$ radial grid. Note the large gap in coverage corresponding to the central $\mathrm{B}$ ring. The lower four panels show the ring $\mathrm{I} / \mathrm{F}$ in three channels: $1.0 \mu \mathrm{m}$ continuum, $2.0 \mu \mathrm{m}$ water ice band, and $3.6 \mu \mathrm{m}$ water ice peak, plus a color composite. The next two panels display the fractional depths (brighter = deeper band) of the water ice bands at 1.25 and $2.0 \mu \mathrm{m}$, while above these is shown the fractional depth of the broad absorption at $0.9-1.8 \mu \mathrm{m}$ which we attribute to $\mathrm{Fe}^{2+}$. The top two panels show color composites assembled from subsets of the lower panels, as labelled on the right edge. Here, blue denotes an ice-dominated region, red a region of strong silicate (?) absorption, and purple a mixture of both.

and on simulations it appears that these particular features are due to light transmitted through the planet's methane-rich atmosphere. The question of whether or not there are detectable amounts of organic material in the rings is thus unresolved at present. We note, however, that small quantities complex organic solids (tholins) mixed with the $\mathrm{H}_{2} \mathrm{O}$ ice of the rings have been invoked to provide the reddish visible coloration of the A and B rings (Cuzzi \& Estrada 1998) and many other Solar System bodies (Cruikshank et al. 2005).

\section{Conclusions}

In the first weeks of the investigation of the Saturn system with VIMS, a number of discoveries have been made, the highlights of which have been presented here. From Earth, Phoebe is a point of light of stellar magnitude 16, but in the VIMS data it has emerged as an icy body of complex and varied surface composition that will eventually yield important information on its origin and history. Water ice is a principal component of its surface. As with the surface materials of Phoebe, the low-albedo material on the leading hemisphere of Iapetus is seen to contain $\mathrm{CO}_{2}$ in some form of chemical or structural complex with the substance(s) imparting the low reflectivity. These two surfaces also share a strong spectral absorption at $3 \mu \mathrm{m}$ attributed to $\mathrm{H}_{2} \mathrm{O}$ bound to mineral grains or organic solid compounds. Both bodies have additional spectral absorption bands that are not yet identified.

High-altitude methane emission is found in the atmosphere of Titan arising from fluorescence at $\sim 400 \mathrm{~km}$. Model calculations indicate a methane abundance of $2 \%( \pm 1 \%)$ at that altitude. Spectral emissions from the night-side atmosphere at $4.3 \mu \mathrm{m}$ and $4.55 \mu \mathrm{m}$ are tentatively identified as $\mathrm{CO}_{2}$ and $\mathrm{CH}_{3} \mathrm{D}$, respectively. Three discrete clouds in Titan's atmosphere near the South Pole were tracked for $13 \mathrm{~h}$, indicating a weak prograde wind with velocity up to $3 \mathrm{~m} \mathrm{~s}^{-1}$.

Circular albedo features on Titan's surface, seen through a window at $2.02 \mu \mathrm{m}$ in the atmospheric $\mathrm{CH}_{4}$ absorption, are suggestive of impact structures that have partially or completely relaxed isostatically in a plastic lithosphere/cryosphere. If these features are indeed remnants of an ancient impacted crust, certain limits to processes of weathering and deposition from the atmosphere can be derived with further study.

With the high spatial resolution and diagnostic spectral coverage afforded by VIMS, the spectral study of Saturn's rings has moved into a new dimension. Small differences in $\mathrm{H}_{2} \mathrm{O}$ ice band strengths in different regions of the ring system are diagnostic of variations in surface grain particle size and regolith scattering properties. The detection of a broad spectral absorption band from $\sim 0.9-1.8 \mu \mathrm{m}$ is suggestive of the presence of ferrous iron-bearing materials in the $\mathrm{C}$ ring and Cassini Division.

Although three previous spacecraft have visited the Saturn system, Cassini is the first to carry a mapping spectrometer that operates in the visual and near-infrared spectral regions. With the diagnostic power provided by a combination of spectral and spatial resolution, VIMS opens a new era in the exploration of Saturn, its rings and icy satellites, and the surface and atmosphere of Titan. 


\section{References}

Baines, K. H., Bellucci, G., Bibring, J.-P., et al. 2000, Icarus, 148, 307 Bellucci, G., D’Aversa, E., Formisano, V., et al. 2004, Icarus, 172, 141 Borderies, N., Goldreich, P., \& Tremaine, S. 1985, Icarus, 63, 406

Boursier, C., Menard, J., Doyennette, L., \& Menard-Bourcin, F. 2003, J. Phys. Chem. A, 107, 5280

Brown, R. H., Baines, K. H., Bellucci, G., et al. 2003, Icarus, 164, 461

Buratti, B. J., Cruikshank, D. P., Brown, R. H., et al. 2005, ApJ, 622, L149

Chamberlain, M. A., \& Brown, R. H. 2004, Icarus, 172, 163

Clark, R. N., Fanale, F. P., \& Gaffey, M. J. 1986, in Satellites, ed. J. A. Burns, \& M. S. Matthews (Univ. of Arizona Press), 437

Clark, R. N., Brown, R. H., Jaumann, R., et al. 2005, Nature, 435, 66

Cruikshank, D. P., Imanaka, H., \& Dalle Ore, C. M. 2005, Adv. Space Res., in press

Cuzzi, J. N., \& Estrada, P. R. 1998, Icarus, 132, 1

Cuzzi, J. N., Estrada, P. R., \& Showalter, M. R. 2003, Icarus, 166, 212

Doyennette, L., Menard-Bourcin, F., Menard, J., Boursier, C., \& Camy-Peyret, C. 1998, Phys. Chem. A, 102, 3849

Drossart, P., Fouchet, Th., Crovisier, J., et al. 1999, in The Universe as Seen by ISO, ed. P. Cox, \& M. F. Kessler, ESA-SP, 427, 169

Durisen, R. H. 1995, Icarus, 115, 66

Durisen, R. H., Cramer, N. L., Murphy, B. W., et al. 1989, Icarus, 80, 136

Durisen, R. H., Bode, P. W., Cuzzi, J. N., Cederbloom, S. E., \& Murphy, B. W. 1992, Icarus, 100, 364

Estrada, P. R., Cuzzi, J. N., \& M. R. Showalter, M. R. 2003, Icarus, 166,21

Geballe, T. R., Kim, S. J., Noll, K. S., \& Griffith, C. A. 2003, ApJ, 583, L39

Goertz, C. K., \& Morfill, G. 1988, Icarus, 74, 325

Griffith, C. A., Owen, T. C., Geballe, T. R., Rayner, J., \& Rannou, P. 2003, Science, 300, 628

Grundy, W. M., Buie, M. W., Stansberry, J. A., Spencer, J. R., \& Schmitt, B. 1999, Icarus, 142, 536
Hapke, B. W. 1981, J. Geophys. Res., 86, 3039

Hibbitts, C. A., Klemaszewski, J. E., McCord, T. B., Hansen, G. B., \& Greeley, R. 2002, J. Geophys. Res., 107, E10, 14

Hibbitts, C. A., Pappalardo, R. T., Hansen, G. B., \& McCord, T. B. 2003, J. Geophys. Res., 108, E5, 2

Holberg, J. B., Forrester, W. T., \& Lissauer, J. J. 1982, Nature, 297, 115

Horn, L. J., \& Cuzzi, J. N. 1996, Icarus, 119, 285

Kim, S. J., Geballe, T. R., \& Noll, K. S. 2000, Icarus, 147, 588

Linn, D. N. C., \& Bodenheimer, P. 1981, AJ, 248, L83

Lissauer, J. J. 1985, Icarus, 62, 433

López-Valverde, M. A., Lellouch, E., \& Coustenis, A. 2005, Icarus, 175,503

McCord, T. B., Carlson, R., Smythe, W., et al. 1997, Science, 278, 271

McCord, T. B., Hansen, G. B., Clark, R. N., et al. 1998, J. Geophys. Res., 103, 8603

McCord, T. B., Coradini, A., Hibbitts, C. A., et al. 2004, Icarus, 172, 104

Miller, E. A., Klein, G., Juergens, D. W., et al. 1996, Proc. SPIE, 2803 , 206

Nicholson, P. D., French, R. G., Tollestrup, E., et al. 2000, Icarus, 145, 474

Owen, T. C., Cruikshank, D. P., Dalle Ore, C. M., et al. 1999, Icarus, 139,379

Owen, T. C., Cruikshank, D. P., Dalle Ore, C. M., et al. 2001, Icarus, 149,160

Porco, C., Nicholson, P. D., Borderies, N., et al. 1984, Icarus, 60, 1

Poulet, F., Cruikshank, D. P., Cuzzi, J. N., Roush, T. L., \& French, R. G. 2003, A\&A, 412, 305

Reininger, F., Dami, M., Paolinetti, R., Pieri, S., \& Falugiani, S. 1996, Proc. SPIE, 2198, 239

Schmit, U., \& Tscharnuter, W. M. 1999, Icarus, 138, 173

Simonelli, D. P., \& Buratti, B. J. 2004, Icarus, 172, 149

Tremaine, S. 2003, AJ, 125, 894

Ward, W. R. 1981, Geophys. Res. Lett., 8, 641 